\title{
Liver Vessel Segmentation Using Gradient Vector Flow
}

\author{
Caspar J. Goch ${ }^{1}$, Xin Wang ${ }^{1}$, Hans-Peter Meinzer ${ }^{1}$, Ingmar Wegner ${ }^{1}$ \\ ${ }^{1}$ Division of Medical and Biological Informatics, DKFZ Heidelberg \\ c.goch@dkfz-heidelberg.de
}

\begin{abstract}
An accurate segmentation of vascular systems is fundamental for many medical applications. Stability against different contrast levels and noise are very important. In this paper we propose an approach for the segmentation of the vascular system of the liver. It is based on the gradient vector flow $(\mathrm{GVF})$ and Frangis vesselness measure. This method avoids multi-scale analysis and related scale space problems. It was evaluated on ten CT data-sets.
\end{abstract}

\section{Introduction}

The segmentation of the vascular tree of the liver is important for diagnosis and therapy. It is the prerequisite for the calculation of supply areas within the liver and segmentation of the liver segments. These are the foundation for surgical planning in the liver.

Many works have been presented for the segmentation of vessels and similar tube-like structures across various organs [1]. Each organ poses different challenges and requires different approaches.

A promising methods is the use of the gradient vector flow technique for segmenting tube-like structures of different scales. Bauer et al. have so far tested it on coronary arteries and bronchial trees [2]. The advantage of the gradient vector flow is a diffusion of the edge information. This removes the need for a multi scale analysis of the image. The range of the edge information is increased, so that different sizes of vessels behave similar during later analysis for vesselness.

This work examines whether this approach can be applied to the segmentation of liver vessels.

\section{Materials and Methods}

The algorithms discussed here are designed to work on already pre-segmented and masked liver data. The liver vessel segmentation is achieved by multiple steps.

For the segmentation of the vascular system of the liver the vessels have to be identified. This is achieved by calculating a vesselness for each voxel. 


\subsection{Preprocessing}

A histogram of the image data is calculated. The maximum of the bell shape is found and the number of voxels with this gray value is saved as $n_{\max }$. This is the non-zero gray value range, that is most prominent in the image. The entire bell shape consists of the gray value ranges of tissue as well as vessels. As vessels are on average brighter than the surrounding tissue, and the vessels comprise a much smaller volume, than the liver tissue in itself, so they do not influence the bell shape distribution in major manner. Trials have shown, that following the bell shape until the number of voxels with a certain gray value drops to under $45 \%$ of $n_{\max }$ leads to a threshold that minimizes the number of of vascular voxels that are missed, while significantly reducing the number of false positives. This value is our lower threshold $t h_{\text {low }}$.

\subsection{Tube Detection}

An image containing the gradient of the original image is calculated

$$
F_{\text {grad }}=\nabla I\left(x_{1}, x_{2}, x_{3}\right)
$$

The resulting image is a vector field, where each vector points to the closest edge.

The image of a masked liver has steep edges around the liver itself. These edges can in the course of our further analysis of the image lead to unwanted responses. To reduce these we suppress any edges between voxel values lower than the $t h_{\text {low }}$ and voxels with a non liver gray value. Typical non liver values are zero in the masked area and values lower than zero if part of the lung has falsely been included in the liver segmentation. Edges between non liver values and values higher than $t h_{\text {low }}$ are not suppressed, as they might belong to a vessel very close to the edge of the segmented area such as vessels joining a vena cava not included in the liver segmentation.

This gradient vector field is then normalized as suggested in the work by Bauer and Bischof [3]. Here normalization is required to account for varying contrast situations. It reduces the influence of very high gradients as introduced by pathologies, such as calcifications. While the reduction of these high responses is desirable a standard normalization would result in unwanted influences by gradients due to noise in the tissue. To prevent this a custom normalization is defined, which gives a standard normalization for high gradients and suppresses low magnitude gradients

$$
F_{n}(x)=\frac{F(x)}{|F(x)|} \frac{\min \left(|F(x)|, F_{\max }\right)}{F_{\max }}
$$

On this vector field the gradient vector flow as proposed by $\mathrm{Xu}$ and Prince [4] is performed.

The vector field $V(x)$ that minimizes the energy for equation (3) is computed 


$$
E(V)=\iiint \mu|\nabla V(x)|^{2}+\left|F_{n}\right|^{2}\left|V-F_{n}\right|^{2} d x
$$

The relevant information is represented in the direction of the resulting vectors while their magnitude is of no importance. Any vectors with an extremely small magnitude are removed. Therefore the resulting vector field is further normalized $V_{n}(x)=V(x) /|V(x)|$. To compute from the vector field the vesselness of structures in the original image we apply an additional gradient filter.

The resulting matrices are similar to the Hessian matrices $H=\nabla * \nabla * I$, but they are not necessarily symmetrical. They are symmetrized by using the following formula

$$
M_{s}(\boldsymbol{x})_{i j}= \begin{cases}M(\boldsymbol{x})_{i j} & i=j \\ \frac{1}{2} \cdot\left(M(\boldsymbol{x})_{i j}+M(\boldsymbol{x})_{j i}\right) & i \neq j\end{cases}
$$

From these matrices a vesselness measure can be calculated. Tube-like bright structures on a darker background can be identified by the eigenvalue of the Hessian matrices. For liver vessels the vesselness measure by Frangi [5] is used.

$$
\nu_{o}= \begin{cases}0 & \text { if } \lambda_{2}>0 \text { or } \lambda_{3}>0 \\ \left(1-\exp \left(-\frac{\mathcal{R}_{\mathcal{A}}{ }^{2}}{2 \alpha^{2}}\right)\right) \exp \left(-\frac{\mathcal{R}_{\mathcal{B}}{ }^{2}}{2 \beta^{2}}\right)\left(1-\exp \left(-\frac{\mathcal{S}^{2}}{2 c^{2}}\right)\right) & \end{cases}
$$

By adjusting the variables $\alpha, \beta, c$ the sensitivity of the vesselness detection can be adjusted.

\subsection{Vesselness Evaluation}

The vesselness image obtained so far results in many non zero responses for structures that are slightly tube like. To reduce such artefacts the vesselness measure is thresholded to include only objects of sufficient similarity to tubular structures.

After reducing the vesselness image to those structures most likely to be bright tubular structures on darker background the vesselness image is considered in combination with the original gray value image. The original gray values of any voxel with a non zero vesselness is compared to the window of likely vessel gray values as calculated during preprocessing. As any tubular structures falling outside of this gray value range are unlikely to be liver vessels they are masked from the vesselness image.

To further reduce spurious responses and isolated false positives the resulting vesselness image is analyzed for connected structures.

These connected areas are then sorted by size and any below a certain size are dismissed as noise. As vascular systems consist of several thousands of voxels a value of 500 as proven to be a reasonable threshold. 


\subsection{Vessel Tree Creation}

Depending on the exact anatomical configuration and whether or not the vena cava is included in the image data the entire vascular tree is detected as a single connected structure or as several. For the detection we assume, that the detected structure with the largest volume is the entire vessel tree. For image data where connecting vessels have been masked out more structures have to be considered to achieve a complete result.

In addition to the segmentation a root point is required for the creation of a vessel tree. This could either be detected automatically or set by the user. For purposes of this work it has been set manually to compare the results with the ground truth data. Using segmentation and root a vessel tree representation of the available data is created as described in [6].

The resulting tree is then pruned to reduce spurious branches:

1. The vessel is very short and does not contain more than two vessel elements.

2. The vessels maximal radius is very small. This indicates either noise, that represents a false vessel or an existing vessel which is very thin. Such vessels are pruned to remove false positives while retaining a highly detailed vessel tree.

3. The vessel has a high mean and maximal angle. Such "corkscrew" shapes are a typical sign of leakage in the segmentation.

\section{Results}

The algorithm described in this work has been evaluated on ten data sets, for each of which a reference tree has been segmented manually by a medical professional. For the evaluation the fixed values of $(\alpha=0.5, \beta=0.5, c=0.5)$ were used, which have been found in a prior evaluation.

Table 1 shows a comparison between the reference trees $\mathrm{R}$ and the computed trees A. The first column is the overall length of all vessels of the computed trees, the second that of the overall length of all vessels of the reference trees. The third and forth column show the length where the centerline of one tree is within the vessel radius of the other. The third column can be read as the specificity, describing which part of the vessel tree is within the boundaries of the reference tree, whereas the fourth is the sensitivity, describing how much of the reference tree is correctly detected.

As can be derived from table 1 the sensitivity ranges between $55 \%$ and $92 \%$ and the specificity between $35 \%$ and $70 \%$. Manual comparison of the corresponding vesseltrees shows, that a likely vessel to be falsely detected is the vena cava, which is correctly segmented in the reference vessels, but seems to be to large and uniform to be correctly detected by the proposed method. Also in those cases where a large part of the reference tree is not within the computed tree, that is due to the vena cava being masked out, resulting in disconnected component trees, so that the second branch is detected as a separate object and discarded before the formation of a vesseltree. 
Table 1. Comparison of reference vessel tree and computed vessel tree (lengths in $\mathrm{mm})$.

\begin{tabular}{lllll}
\hline Case & Length A & Length R & Length A in R & Length R in A \\
\hline F & 3768.04 & 5869.52 & $2266.09(60 \%)$ & $3996.76(68 \%)$ \\
G & 4521.36 & 2312.44 & $1611.73(36 \%)$ & $2144.90(93 \%)$ \\
H & 6023.04 & 4910.82 & $2426.61(40 \%)$ & $3507.99(71 \%)$ \\
I & 5594.76 & 3831.89 & $2096.14(37 \%)$ & $3077.99(80 \%)$ \\
J & 3418.08 & 6119.70 & $2072.29(61 \%)$ & $3419.02(56 \%)$ \\
K & 4693.68 & 5228.94 & $3078.57(66 \%)$ & $4184.73(80 \%)$ \\
L & 5569.00 & 7319.06 & $2790.02(50 \%)$ & $4961.20(68 \%)$ \\
M & 6878.02 & 5332.84 & $2961.60(43 \%)$ & $4162.16(78 \%)$ \\
N & 6222.01 & 6164.64 & $3700.52(59 \%)$ & $4840.51(79 \%)$ \\
O & 6790.90 & 7871.08 & $4414.51(65 \%)$ & $6658.95(85 \%)$ \\
\hline
\end{tabular}

\section{Discussion}

The presented work shows that the gradient vector flow technique can be used to segment the vascular system of the liver using the gradient vector flow approach. By using this method the computationally very intensive multi scale calculations can be avoided.

Acknowledgement. This work was supported by the German Research Foundation (DFG) in the context of the research project "Protektive Beatmungskonzepte".

\section{References}

1. Lesage D, Angelini ED, Bloch I, et al. A review of 3D vessel lumen segmentation techniques: models, features and extraction schemes. Med Image Anal. 2009;13:819 45 .

2. Bauer C, Bischof H, Beichel R. Segmentation of airways based on gradient vector flow. In: Proc EXACT; 2009. p. 191-201.

3. Bauer $\mathrm{C}$, Bischof $\mathrm{H}$. Edge based tube detection for coronary artery centerline extraction. Insight J. 2008.

4. Xu C, Prince JL. Snakes, shapes, and gradient vector flow. In: IEEE Trans Image Process. vol. 7; 1998. p. 359-69.

5. Frangi AF, Niessen WJ, Vincken KL, et al. Multiscale vessel enhancement filtering. In: Proc MICCAI; 1998. p. 130-7.

6. Schöbinger M, Thorn M, Vetter M, et al. Robuste Analyse von Gefäßstrukturen auf Basis einer 3D-Skelettierung. In: Proc BVM; 2003. p. 76-80. 